\title{
Teorema do Confronto: discussão didática alternativa articulando as práticas usuais e o software Geogebra
}

\author{
Squeeze Theorem: alternative didactical discussion articulating usual \\ practices and the Geogebra software
}

\author{
Pedro Mateus* \\ ORCID iD 0000-0002-6523-126X \\ Marlene Alves Dias** \\ ORCID iD 0000-0001-9168-9066
}

\begin{abstract}
Resumo
Neste artigo, visamos determinar as relações pessoais dos estudantes do $1^{\circ}$ ano do curso de licenciatura em ensino de Matemática, em Moçambique, sobre o teorema do confronto e a eficácia didática do software Geogebra na discussão desse teorema na sala de aula. As perguntas da pesquisa foram: como os estudantes participantes do experimento compreendiam o teorema do confronto e como o Geogebra capitalizaria a discussão desse teorema na sala. Usamos a Teoria Antropológica do Didático e a Teoria de Instrumentação. A pesquisa foi de natureza qualitativa, na forma do estudo de caso. Estrategicamente, fizemos: análise preliminar e a priori do conteúdo visado, o experimento, a análise a posteriori e validação interna dos resultados. $\mathrm{O}$ experimento possibilitou compreender como o uso do computador, articulado com as práticas usuais, pode auxiliar a compreensão dos estudantes sobre o teorema do confronto.
\end{abstract}

Palavras-chave: Mediação Didática. Teoria Antropológica do Didático. Teoria de Instrumentação.

\begin{abstract}
In this article, we aim at determining the personal relationship of $1^{\text {st }}$-year Mozambican undergraduate prospect teachers in mathematics with the Squeeze Theorem and the didactical effectiveness of Geogebra in its discussion in the classroom. The research question was: how did the students, who participated in the experiment, understand the Squeeze Theorem and how would the Geogebra software capitalize the discussion of this theorem in the classroom? We used the Anthropological Theory of Didactics and the Theory of Instrumentation. It was a qualitative research in the shape of a case study. Strategically, we did a preliminary and a priori analysis of the target content, the experiment itself, a posteriori analysis and internal validation of the results. The experiment allowed us to understand how the blended teaching through the computer and usual practices can assist students in understanding the Squeeze Theorem.
\end{abstract}

Keywords: Didactical mediation; Anthropological theory of didactics; Instrumentation theory.

\footnotetext{
* Doutor em Educação Matemática pela Universidade Anhanguera de São Paulo (UNIAN). Professor na Universidade Pedagógica - Delegação da Beira (UP - Beira), Beira, Sofala, Moçambique. Endereço para correspondência: Rua Dom Francisco de Almeida, 316-R/C, Ponta-Gêa, Beira, Sofala, Moçambique, CP: 2025. E-mail: pmateuszulu @gmail.com.

** Doutora em Matemática pela Universidade Dennis Diderot - Paris 7, França. Professora do Programa de Pósgraduação da Universidade Anhanguera de São Paulo (UNIAN), São Paulo, Brasil. Endereço para correspondência: Rua Sobralia, 407, São Paulo, São Paulo, Brasil, CEP: 04691-020. Email: maralvesdias@gmail.com.
} 


\section{Introdução}

Ficamos motivados para discutir neste trabalho o teorema do confronto pela sua característica pouco clara no que tange ao raciocínio dedutivo implicado, o qual apresenta um nível de abstração de difícil compreensão pelos estudantes, impedindo-os de usá-lo corretamente nas atividades matemáticas em que o teorema é requerido. Esse teorema é um dos clássicos problemas de análise matemática, aparecendo quase sempre em publicações dessa área de conhecimento, por um lado, e motivo de inquietação didática na sala de aula, por outro. Para dar exemplos dessa inquietação, destacamos a pesquisa de Hitt (2011), intitulada "Construction of mathematical knowledge using graphic calculators (CAS) in the mathematics classroom" com estudantes canadenses em formação para serem professores do Ensino Médio, na qual encontramos a tarefa apresentada no Quadro 1:

Considere a função definida por $f(x)=\left\{\begin{array}{ll}x^{2} \operatorname{sen}\left(\frac{1}{x}\right) & \text { se } x \neq 0 \\ 0 & \text { se } x=0\end{array}\right.$. Encontre a função derivada de fe seu valor (da função derivada) no ponto $x=0$.

Quadro 1 - Tarefa Teorema do Confronto

Fonte: Hitt (2011, p. 723)

Na resolução efetuada pelos estudantes canadenses da tarefa acima, percebemos a real complexidade do teorema do confronto, pois as calorosas e longas discussões entre os formandos não conduziram a um consenso no sentido de descobrir se a função dada era ou não diferenciável no ponto dado $\mathrm{x}=0$. Alguns entre os estudantes envolvidos na discussão achavam que função dada era diferenciável no ponto considerado, mas não foram capazes de apresentar argumentos plausíveis que justificassem a resposta. Os argumentos plausíveis, neste caso, seriam a caracterização da função razão incremental e a evocação do teorema do confronto, pois ela (função razão incremental) reúne as premissas desse teorema. Os estudantes que apresentavam a opinião contrária arguíam que a função dada aparentava uma descontinuidade em $\mathrm{x}=0$, mas sem caracterizar precisamente a natureza dessa descontinuidade.

Antes da leitura do estudo de Hitt (2011), não estávamos conscientes do que realmente poderia acontencer em termos do desenvolvimento do raciocínio matemático dos estudantes diante dessa tarefa. Depois de termos lido essa discussão, consideramos experimentar e discutir a mesma tarefa com um grupo de sete estudantes voluntários do $1^{\circ}$ ano 
do curso de licenciatura em ensino de Matemática da Universidade Pedagógica, no campus da Beira, em Moçambique, procurando verificar como eles reagiriam à tarefa e de que forma a incorporação do recurso computacional Geogebra seria uma opção didática complementar para alimentar a discussão desse teorema.

Assim, escolhemos como referencial teórico da pesquisa a Teoria Antropológica do Didático de Chevallard - TAD (CHEVALLARD, 1999, 2014) e a teoria de instrumentação de Rabardel (RABARDEL, 1995, 2002), que nos ajudaram a identificar de forma mais clara os objetivos da pesquisa, a metodologia, a interpretação e justificação dos dados encontrados. Na TAD, consideramos as noções de praxeologia, objetos ostensivos e não ostensivos na atividade matemática e ecologia dos saberes matemáticos. A teoria de instrumentação deu-nos suporte para refletir sobre o significado da relação que o pesquisador e os estudantes foram construindo na interação com as tarefas e com o computador durante as discussões nas sessões experimentais. Destacamos que esse estudo é parte de uma pesquisa de doutorado por nós desenvolvida em 2014, intitulada "Derivadas de funções reais de uma variável real e integral de Riemann: construção e aprendizagem de conceitos mediadas por mídias e práticas usuais". Adiante, destacamos resumidamente as duas teorias escolhidas para realizar o estudo proposto.

\section{Teoria Antropológica do Didático}

A Teoria Antropológica do Didático - TAD enquadra a atividade matemática no conjunto das atividades humanas regularmente desenvolvidas, descrevendo o conhecimento matemático em termos de organizações praxeológicas ou praxeologias $\wp$ cujas noções básicas são as noções de tipos de tarefas $\mathrm{T}$, técnicas $\tau$ (maneiras de resolver as tarefas de um dado tipo), tecnologias $\theta$ (um discurso racional visando justificar, explicar e produzir técnicas) e teorias $\Theta$ (objetivando justificar, explicar e produzir tecnologias) que possibilitam modelar as práticas sociais em geral e as atividades matemáticas, em particular, conforme Chevallard $(1999,2014)$. A partir desse ponto de vista, segundo o autor, a praxeologia $\wp$ consiste de um bloco prático técnico, П (praxis) [tipos de tarefas $(\mathrm{T}) /$ técnicas $(\tau)$ ], que corresponde a um saber fazer, e um bloco tecnológico-teórico, $\Lambda(\log o s)$ [tecnologia $(\theta)$ /teoria $(\Theta)$ ], que corresponde a um saber.

Segundo Chevallard (2014, 1999), a noção de tipo de tarefas pressupõe um objeto relativamente preciso, para o qual existe uma técnica $\tau$ acompanhada de um discurso 
tecnológico-teórico mais ou menos preciso. Em muitos casos, um tipo de tarefas se expressa por meio de um verbo evocando uma ação, o que há para fazer. Por exemplo, derivar a função $f(x)= \begin{cases}x^{2} \operatorname{sen}\left(\frac{1}{x}\right) & \text { se } x \neq 0 \\ 0 & \text { se } x=0\end{cases}$ no ponto $\mathrm{x}=0$ é um tipo de tarefas que pode ser resolvido com a técnica do Teorema do Confronto, e ao mesmo tempo justificada com a tecnologia associada ao mesmo teorema no contexto da Teoria de Limites de Funções.

Como exemplo da teoria, apresentamos na figura 1 a formulação de Guidorizzi (2008) desse teorema e dois exemplos de sua aplicação:

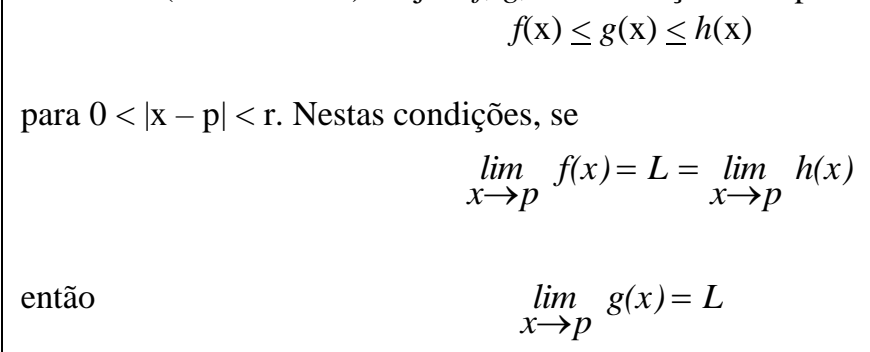

$$
\lim _{x \rightarrow p} f(x)=L=\lim _{x \rightarrow p} h(x)
$$

então

$$
\lim _{x \rightarrow p} g(x)=L
$$

$[\ldots]$

Exemplo 2: Sejam $f$ e $g$ duas funções com mesmo domínio $A$ tais que $\lim _{x \rightarrow p} f(x)=0$ e $|g(x)| \leq M$ para todo $x$ em $A$, onde $\mathrm{M}>0$ é um número real fixo. Prove que $\lim _{x \rightarrow p} f(x) g(x)=0$

Solução

$$
|f(x) g(x)|=|f(x)||g(x)| \leq M|f(x)|
$$

para todo $x$ em $A$. Daí, para todo $x$ em $A$

$$
-M|f(x)| \leq f(x) g(x) \leq M|f(x)|
$$

De $\lim _{x \rightarrow p} f(x)=0$ segue que $\lim _{x \rightarrow p} M|f(x)|=0$ e $\lim _{x \rightarrow p}-M|f(x)|=0$. Pelo teorema de confronto

$$
\lim _{x \rightarrow p} f(x) g(x)=0
$$

$[\ldots]$

Exemplo 3. Calcule $\lim _{x \rightarrow 0} x^{2} g(x)$ onde $g(x)=\left\{\begin{array}{c}1 \text { se } x \in Q \\ -1 \text { se } x \notin Q\end{array}\right.$

Solução

$\lim _{x \rightarrow 0} x^{2}=0$; como $\lim _{x \rightarrow 0} g(x)$ não existe (verifique) não podemos aplicar a propriedade relativa a limite de um produto de funções. Entretanto, como $g$ é limitada, $(|g(x)| \leq 1$ para todo $\mathrm{x}) \mathrm{e}$

$$
\lim _{x \rightarrow 0} x^{2}=0 \text {, pelo exemplo anterior } \quad \lim _{x \rightarrow 0} x_{0}^{2} g(x) ;=0 \text {. }
$$

Figura 1 - Teoria do Confronto e sua aplicação Fonte: Guidorizzi (2008, p. 90-92) 
É particularmente interessante observar que a opção do autor, de acordo com a figura acima, assim como seu âmbito de aplicação, não fazem apelo a nenhuma intuição gráfica, o que consideramos que pode ser uma fonte adicional de dificuldade para um aprendiz que ainda pode estar dependente de alguns elementos intuitivos de referência para compreender o teorema em sua plenitude. Obervamos ainda a importância dos exemplos que o autor apresenta, pois eles mostram o âmbito de aplicação do teorema.

Contudo notamos a ausência do discurso tecnológico associado à demonstração do teorema que seria importante ter sido apresentado para primeiro prover uma justificativa no sentido de que, segundo Chevallard $(2014,1999)$, essa prática leva-nos ao resultado desejado e que é correto proceder dessa maneira. Pensamos que esse discurso pode ser melhor compreendido quando lhe damos um suporte, por meio da representação gráfica, que auxilia a fundamentar as propriedades utilizadas na demonstração do teorema e dos exemplos considerados, pois ao utilizar o ostensivo escrito Teorema do Confronto para demonstrar que o limite do produto das funções $\mathrm{f}$ e $\mathrm{g}$ quando $\mathrm{x}$ tende a $\mathrm{p}$ é igual a zero, estamos nos referindo ao não ostensivo Teorema do Confronto, que corresponde ao conceito que lhe é institucionalmente associado.

Dessa forma, a necessidade de prover alguns elementos intuitivos no processo de aprendizagem do teorema do confronto nos conduziu a incorporar nas atividades por nós denominadas experimentais a representação gráfica de algumas etapas da análise desse teorema, pois, por meio do ostensivo gráfico, podemos auxiliar os estudantes a criarem imagens mentais importantes para a compreensão do teorema e de sua aplicação.

Consideramos que essa opção didática é pertinente, quando nos propomos a analisar a atividade matemática na perspectiva da TAD, pois segundo Chevallard (2002), as condições e restrições que permitem a produção e o uso de tarefas matemáticas nas diferentes instituições (a ecologia de uma praxeologia matemática) dependem dos objetos ostensivos, aqueles perceptíveis aos sentidos humanos e capazes de serem manipulados, tais como os sons, gráficos, gestos, escritas e dos objetos não ostensivos que correspondem aos conceitos, noções e ideias que podem ser evocados quando se manipulam os ostensivos que lhes são associados. Para tal, enquanto opção didática que possibilita uma discussão por meio da dialética ostensivos e não ostensivos, assumimos o Geogebra como um objeto ostensivo que torna possível materializar e manipular as noções, conceitos e ideias associadas ao Teorema do Confronto.

Reiteramos ainda, citando Chevallard (2002), que os objetos não ostensivos não podem ser vistos, percebidos ou mostrados por si mesmos. Assim, eles só podem ser 
evocados por meio de uma manipulação adequada de certos objetos ostensivos e estes últimos dependem das escolhas que lhe são associdas nas diferentes instituições. Por exemplo, para determinar a derivada da função como a dos exemplos acima considerados, evocamos algumas ideias, princípios e leis de derivação que não podem ser vistas, mas elas guiam as ações da pessoa que a usa para obter o resultado pretendido, como a solução proposta para o exemplo 3, que utiliza as propriedades de limite para as funções dadas a fim de encontrar o resultado do limite do produto dessas funções, quando x tende a zero. O exemplo coloca em evidência a dialética ostensivos e não ostensivos necessária para o desenvolvimento desta tarefa, cuja tecnologia está associada à teoria que a sustenta.

Assim, justificamos nossa escolha do software Geogebra, como um ostensivo rico na materialização dos objetos não ostensivos que constituem os conceitos associados à noção de derivada de funções reais a valores reais.

Para melhor compreender o papel do software Geogebra no desenvolvimento da nossa pesquisa, consideramos a teoria de instrumentação de Rabardel (1995, 2002), para a qual apresentamos na sequência algumas ideias que serviram de apoio ao trabalho por nós desenvolvido.

\section{Teoria de instrumentação}

Destacamos alguns aspetos da teoria de instrumentação/instrumentalização na perspectiva de Rabardel (1995, 2002), tendo em conta sua importância para a análise das relações entre o pesquisador e os estudantes nos seus esforços de interação com as tarefas propostas, mediadas pelo computador durante as discussões nas sessões experimentais.

Para Rabardel (1995, 2002), o instrumento está no coração da atividade humana, ele substitui algumas funções (mentais) pelas outras e reconstrói toda a estrutura do comportamento.

Ao propormos o Geogebra, pressentimos e imaginamos que esse software modifica as práticas de ensino e de aprendizagem do Cálculo Diferencial e Integral, em geral, e da diferenciação, em particular, pois em função do aprimoramento da articulação entre as representações algébricas e gráficas, é possível criar imagens mentais importantes que auxiliam na abstração e generalização de conceitos e noções associados ao Cálculo Diferencial e Integral.

Assim, iniciamos ressaltando que, consoante Rabardel (1995, 2002), a posição intermediária do instrumento faz dele um mediador das relações entre o sujeito e o objeto 
finalizado. É um mundo intermediário, cuja característica principal é adaptar-se, tanto ao sujeito, quanto ao objeto. Para o autor, essa adaptação ocorre em termos materiais e em termos das propriedades cognitivas e semióticas, de acordo com o tipo de atividade na qual o instrumento é inserido ou é supostamente inserido. Dessa forma, segundo o autor, dois tipos de mediação são identificados:

- mediação do objeto ao sujeito, descrita como sendo uma mediação epistêmica, na qual o instrumento é meio que permite ao usuário conhecer o objeto pretendido. Diante dessa mediação, as retroações que a interface emite para o sujeito provocam nele imagens visuais ou acústicas que vão se reestruturando na sua mente em conceitos, objeto finalizado da mediação.

- uma mediação pragmática, do sujeito ao objeto, na qual o instrumento é um meio para ação transformadora, num sentido mais amplo, incluindo controle e regulação, dirigida ao objeto.

A elaboração instrumental pelo usuário é, assim, dirigida tanto para si mesmo, sendo esta a dimensão da gênese instrumental chamada instrumentação, quanto para o artefato, que é a dimensão da gênese instrumental denominada instrumentalização. Assim sendo, destacamos abaixo dois processos associados considerados pelo autor como estando na gênese instrumental.

Processos de instrumentação. Estes processos estão relacionados com a emergência e evolução do uso de esquemas e ações mediadas pelo instrumento: sua constituição, seu funcionamento, sua evolução por acomodação, combinação, coordenação, inclusão e assimilação mútua, assimilação de novos artefatos ao conjunto de esquemas já existentes.

Processos de instrumentalização. Estes processos estão relacionados com a emergência e evolução de componentes artefatuais do instrumento: seleção, consolidação, produção e instituição de funções, desvios e catacreses, atribuição de propriedades, transformação artefatual (estrutura e função, entre outras) que prolongam as criações e ganhos dos artefatos. Após apresentação de algumas ideias subjacentes à Teoria de Instrumentação, destacamos a seguir as considerações metodológicas do estudo.

\section{Método da pesquisa}

A pesquisa foi de natureza qualitativa na forma do estudo de caso, segundo Lüdke e André (1986): 
- tem o ambiente natural como sua fonte de dados e o pesquisador como seu principal instrumento;

- os dados coletados são predominamente descritivos;

- a preocupação com o processo é muito maior do que com o produto;

- o significado que as pessoas dão às coisas é foco de atenção especial pelo pesquisador;

- a análise dos dados tende a seguir um processo indutivo.

Em termos de estratégias, para realizarmos a pesquisa, operacionalizamos alguns elementos da engenharia didática (ARTIGUE, 2010), mais especificamente: análise institucional, concepção e análise a priori das tarefas preparadas para a experimentação; experimentação; análise a posteriori e validação interna dos resultados. Neste artigo, em razão do espaço disponível limitado, destacamos apenas os resultados referentes à experimentação, análise a posteriori e validação interna para a tarefa relacionada ao teorema do confronto.

Essa tarefa foi discutida na $10^{\mathrm{a}}$ sessão das 12 sessões que compreenderam o experimento, no dia 08 de junho de 2013, com uma duração de cerca de 1 hora e 30 minutos, no horário das 10h30min às 12 horas, e participaram da discussão sete estudantes dos oito que regularmente faziam parte do estudo. As discussões decorriam na sala de informática, com cerca de 40 computadores operacionais e os estudantes trabalhavam em duplas, sendo um computador para cada dupla, com o objetivo de propiciar discussões entre os componentes de cada dupla durante a resolução das tarefas propostas pelo pesquisador. Na sequência, os estudantes discutiam no grupo, sob a mediação do pesquisador, os resultados encontrados. Finalmente, os resultados eram validados e a noção ou o conceito em jogo institucionalizado. Usamos duas categorias de análise dos dados, abstraídas do referencial teórico estabelecido:

Categoria A: tipo de relações pessoais dos estudantes participantes da sessão diante das relações institucionais vigentes sobre o Teorema do Confronto;

Categoria B: alcance da mediação didática com recurso ao software Geogebra sobre o Teorema do Confronto.

A seguir destacamos alguns extratos de como a experimentação foi conduzida.

No início, o pesquisador entregou aos estudantes o formulário com as tarefas para resolver. Aqui apresentamos apenas a tarefa que será analisada neste artigo. 
Tarefa 6: Função derivada.

Considere a função f definida por: $f(x)= \begin{cases}x^{2} \operatorname{sen}\left(\frac{1}{x}\right), & \text { se } x \neq 0 \\ 0 \quad & \text { se } x=0\end{cases}$

Encontre a função derivada de $f$.

Estude a derivabilidade de f no ponto $x=0$. Justifique a sua resposta

Figura 2 - Tarefa proposta aos estudantes para derivar Fonte: O autor (2014)

Em seguida, o pesquisador recomendou que os estudantes resolvessem em duplas a tarefa proposta.

A seguir, apresentamos algumas passagens da discussão e a análise da tarefa 6 .

\section{Os resultados e análises da tarefa 6}

Depois de cerca de 30 minutos de discussão entre os estudantes, surgiram alguns resultados que apresentamos a seguir:

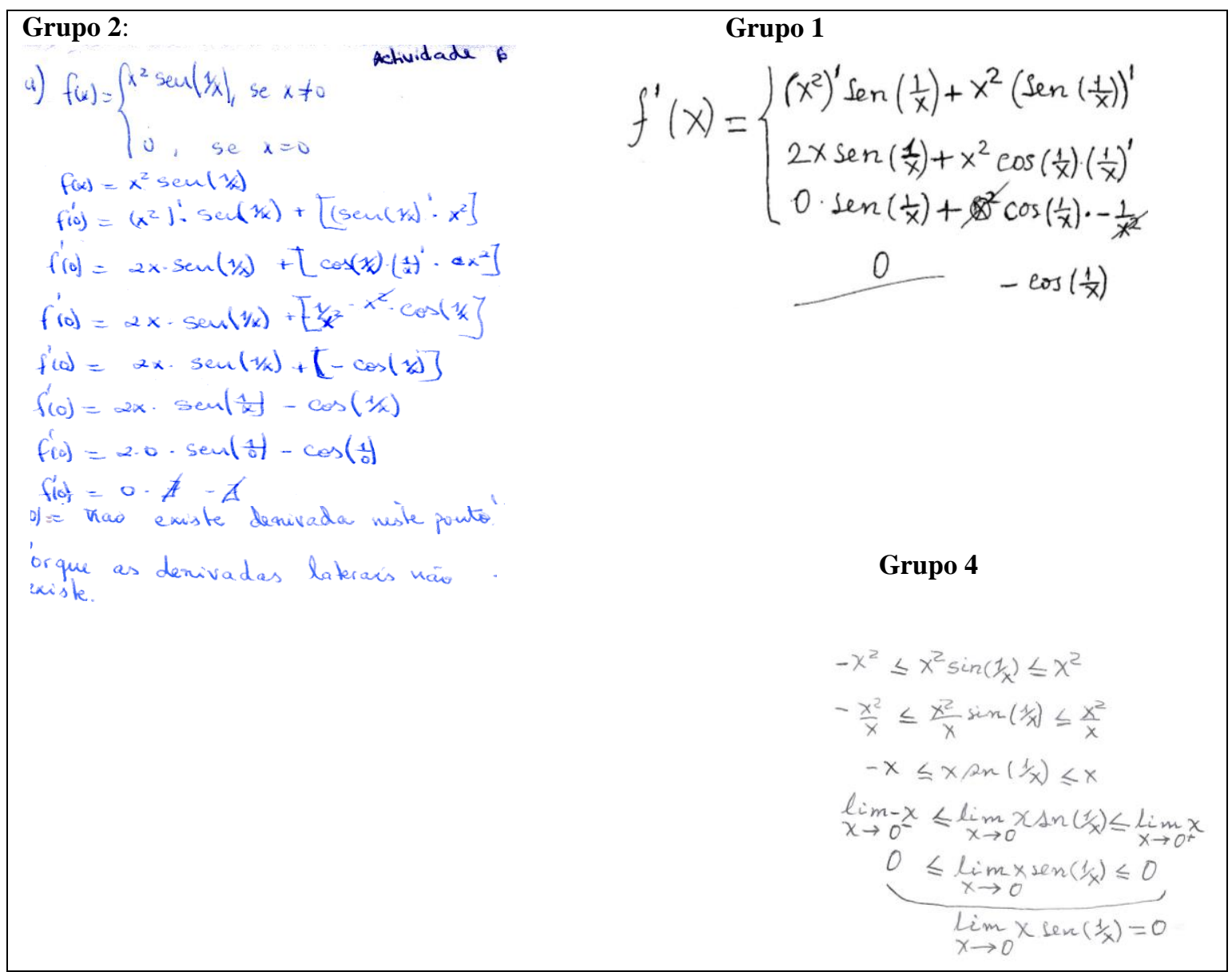

Figura 3 - Derivação de uma função composta pelo Teorema do Confronto Fonte: $\mathrm{O}$ autor (2014) 
Os resultados acima sinalizam que os grupos 1 e 2 não discriminaram bem as condições de definição da função dada por intervalos e, por isso, todas as justificativas por eles apresentadas não eram adequadas, embora a aplicação do Teorema da Função Composta tenha sido bem interpretada. Por outro lado, notamos nas respostas dos grupos referidos o efeito da técnica de substituição aplicada no cálculo de limites, conduzindo o estudante a interpretar o limite da função em um ponto como sendo seu valor nesse ponto, o que não é correto, conforme a própria definição do conceito de limite. Em termos de ecologia matemática para esses estudantes, segundo Chevallard (2002), diríamos que existe uma adaptação inadequada às restrições e às condições da tarefa proposta. As suas relações pessoais não são conformes às relações institucionais vigentes sobre o teorema do confronto.

Já o grupo 4 refletiu convenientemente e deu uma resposta conceitualmente bem justificada, baseada no Teorema do Confronto. Pela nossa experiência, podemos afirmar que muitos estudantes do Ensino Médio e mesmo do Ensino Superior em Moçambique não estão habituados a elaborar respostas desse tipo, baseadas em conceitos. O que eles normalmente produzem são respostas respaldadas em técnicas, fato compreensível, uma vez que os estudantes apresentam uma sequência de manipulação de ostensivos sem evocar os não ostensivos que lhes são associados. No caso, mesmo o grupo 4, ao utilizar o Teorema do Confronto, não explicita qual é o não ostensivo em jogo. Trata-se de uma maneira de trabalhar com a Matemática que não é característica da própria Matemática, mas do ensino de Matemática em alguns países, em particular, em Moçambique e no Brasil, como foi possível constatar pela nossa experiência no caso de Moçambique e por meio do trabalho de Ávila (2005), no qual se destaca que os objetivos do primeiro curso de Cálculo estão prioritariamente associados ao provimento de técnicas.

Parece-nos importante a discussão tecnológico-teórica dessas técnicas, que além de auxiliar a compreendê-las, poderá também ser fonte para a introdução e desenvolvimento de novas técnicas. Assim, consideramos que, embora o ponto de vista de ênfase às tecnicas seja pertinente na perspectiva do autor, é importante que estas técnicas não apareçam do nada, mas sejam alicerçadas em algum discurso tecnológico-teórico que as torne exequíveis, transparentes, justificáveis e explicáveis.

Essa nossa observação é também sustentada por Chevallard (1999) e Bosch, Fonseca e Gascón (2004) de modo que, segundo eles, uma técnica deve ser compreensível, legível e justificável para proporcionar o seu controle e garantir a eficácia das tarefas que ela permite realizar. Ainda de acordo com os mesmos autores, em qualquer instituição $I$, qualquer que seja o tipo de tarefas $T$, a técnica $\tau$ relativa a $T$ está sempre acompanhada de ao menos um 
embrião, ou mais frequentemente dito, de um vestígio da tecnologia $\theta$. Portanto, como já indicado, não é interessante que se apliquem técnicas de substituição, como os estudantes acima fizeram, sem justificativas e explicações do que é feito.

Desse modo, perante as dificuldades como as mostradas pelos grupos 1 e 2, consideramos usar a ferramenta computacional para tentar discutir os equívocos. Nos extratos abaixo, tentamos resumir essa discussão que, na prática, foi muito longa.

$\mathrm{Na}$ Figura 4, que corresponde à discussão do grupo dos estudantes com a mediação do professor, após os trabalhos em duplas, os estudantes estão indicados com os códigos 1A, As, AAs, N, G........e o professor com o código $\mathbf{P}$, com o significado de: $\mathbf{1 A}$ - um estudante; As Os estudantes (geralmente em coro), AAs - alguns estudantes; $\mathbf{N}$ - estudante de nome N; $\mathbf{G}$ estudante de nome G, e assim por diante.

1A - Na $1^{\text {a }}$ tarefa usamos o seletor. A mesma ideia pode ser usada aqui. Sim, pode ser usada. $\mathbf{P}$ - Pode ser usada, é uma possibilidade. Então vamos usar. Para poder usar o seletor, devo ter um ponto no qual devo traçar a reta tangente e determinar o declive da reta tangente naquele ponto.

Os estudantes juntamente com o pesquisador fazem o seguinte desenho:

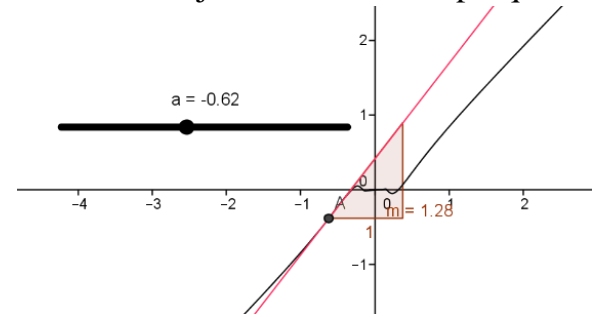

$\mathbf{P}$ - Temos lá o gráfico de $f$, a reta tangente no ponto $A$, e o declive da reta tangente no ponto A. Ou também, como vimos, chamamos de taxa de variação, não é? É a variação da ordenada para a variação de uma unidade na abscissa. O que acontece movimentando o ponto A?

As - Declive altera.

$\mathbf{P}$ - Estamos nas proximidades de zero, qual é a inclinação da reta tangente aqui? (Veja que a tradução de Stewart usa mais o termo inclinação da reta tangente).

AAs - Zero.

$1 \mathbf{A}$ - Não existe.

$\mathbf{P}-\mathrm{Hã}$ ?

AAs - Ali está zero.

$\mathbf{P}$ - Bem, é mudar a cor da reta tangente para ver bem. (O pesquisador sugere a um estudante que não destacou bem no seu desenho). Mude a cor da reta tangente para vermelho (Cor foi uma variável muito importante para distinguir os objetos nessa experimentação). Talvez aumentar um pouco o tamanho da reta tangente.

$\mathbf{P}$ - Este é o $a$, (aponta o $a$ a indicar zero, $\mathrm{a}=0$ ), qual é o declive da reta tangente?

As - Zero. A reta tangente está sobre o eixo das abscissas.

$\mathbf{P}$ - Quer dizer, nas proximidades de zero existe a reta tangente, e mesmo no zero. E mesmo no zero, então o que há a dizer?

N - É derivável no ponto zero. A derivada é zero. Mas calcular analiticamente, vai sair?

Figura 4 - Derivação de uma função composta pelo Teorema do Confronto: discussão Fonte: O autor (2014) 
Até o momento em que essa tarefa foi discutida, os estudantes já sabiam usar os recursos básicos do Geogebra: inserção de funções na entrada de comandos, uso do controle deslizante para desencadear animações, produção de reta tangente num ponto do gráfico de uma função e incorporação do recurso declive na reta. Convém indicar ainda que na sessão anterior a esta em que discutimos o Teorema do Confronto, analisamos os diferentes pontos de vista da derivada, segundo Thurston (1995):

1. Como infinitesimal: a derivada como uma relação de mudança infinitesimal do valor da função à mudança infinitesimal da variável;

2. no sentido simbólico: a derivada como resultado de uma certa manipulação simbólica. A derivada de $\mathrm{x}^{\mathrm{n}}$ é $\mathrm{nx}^{\mathrm{n}-1}$, a derivada de $\operatorname{sen}(\mathrm{x})$ é $\cos (\mathrm{x})$, a derivada de fog é f'ogog' etc.

3. no sentido lógico: a derivada como objeto definido sob certas condições lógicas. $f^{\prime}(\mathrm{x})=d$, se e somente se, para cada $\varepsilon$, existe um $\delta$ tal que se

$0<|\Delta x|<\delta$ então $\left|\frac{f(x+\Delta x)-f(x)}{\Delta x}-d\right|<\varepsilon$

4. no sentido geométrico: a derivada como o declive da reta tangente ao gráfico de $f$ em um ponto $P$ dado, se o gráfico tiver uma reta tangente nesse ponto;

5. taxa de variação: a derivada como taxa de variação instantânea de $f(\mathrm{t})$ se $t$ for o tempo;

6. uma aproximação: a derivada como aproximação linear de uma função $f$ nas proximidades do ponto A dado;

7. do ponto de vista microscópico: a derivada de uma função como o limite do que se vê sob um microscópio ampliando cada vez mais (THURSTON, 1995, p. 10, tradução nossa).

Com recurso ao software Geogebra, consideramos que a discussão da existência ou não da derivada da função dada no ponto $\mathrm{x}=0$ foi efetiva. Visualmente foi observado e justificado que a derivada da função dada, no ponto $x=0$, era zero. Nesse contexto, pensamos que as possibilidades de validação visual, experimentação e indução à formulação de conjecturas, proporcionadas pelo software Geogebra, sejam alguns dos grandes méritos didáticos do Geogebra em Matemática. Os argumentos dos estudantes foram conceitualmente coerentes. O questionamento do estudante N: “... Mas calcular analiticamente vai sair?” achamos ser de grande valor didático para capitalizá-lo rumo à elaboração do discurso técnico tecnológico do teorema do confronto.

Como encaminhar o discurso algébrico, articulando-o com a representação gráfica?

O extrato seguinte mostra essa tentativa: 


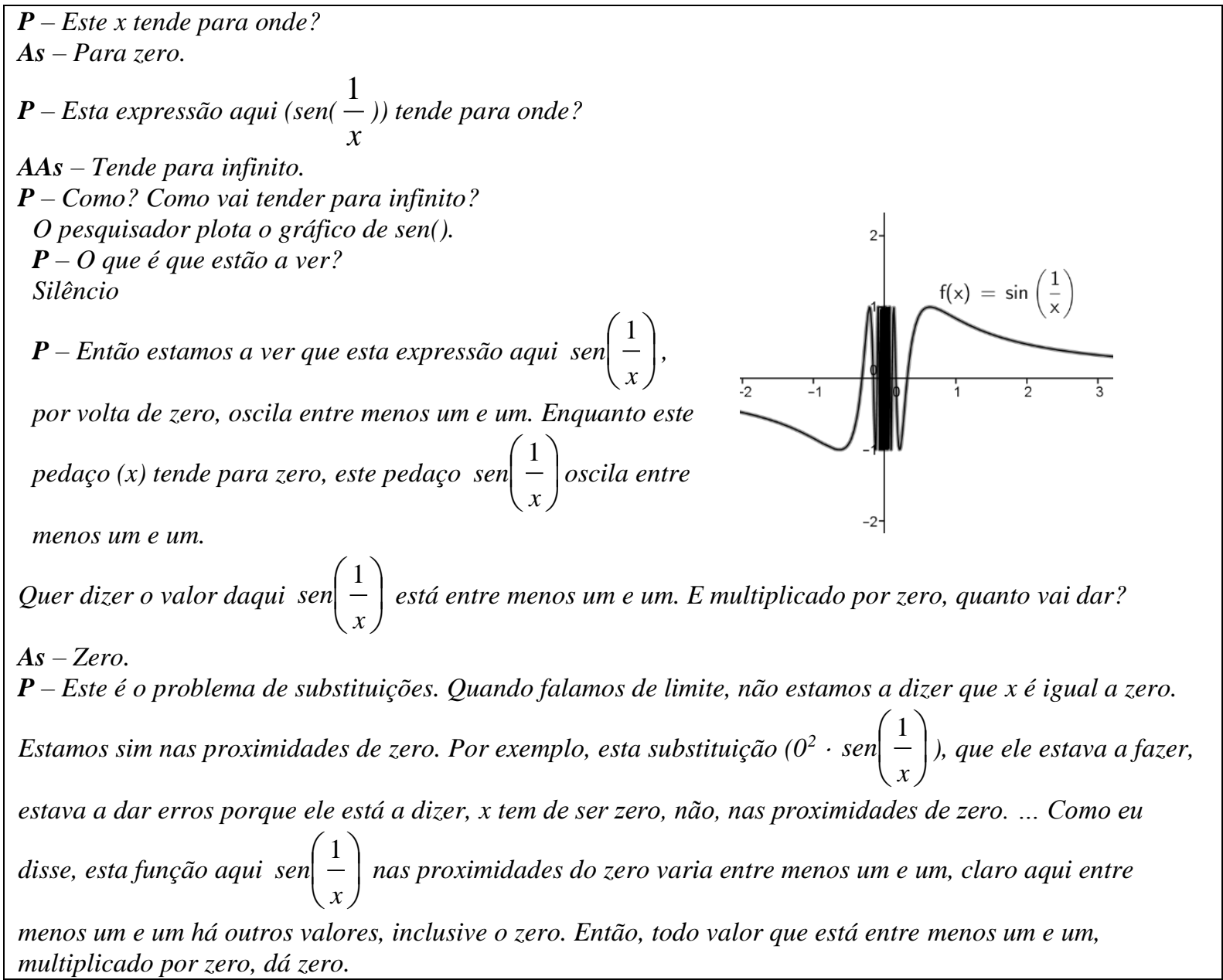

Figura 5 - Análise com auxílio da representação gráfica do Teorema do Confronto Fonte: O autor (2014)

Percebemos, na figura acima, parte do decurso da sessão, na qual vemos a complexidade do estudo da derivada da função $f(x)=\left\{\begin{array}{ll}x^{2} \operatorname{sen}\left(\frac{1}{x}\right), & \text { se } x \neq 0 \\ 0 \quad, & \text { se } x=0\end{array}\right.$ no ponto $x=0, \mathrm{e}$ as reais dificuldades que surgem e que estão intimamente relacionadas com a definição do conceito de limite, quando se generaliza por meio da técnica de substituição.

Não se sabe ao certo como essa técnica é construída no meio estudantil, porque institucionalmente não é destacada. Todas as definições de limites de uma função em um ponto apontam que o número $a$ para o qual determinamos o limite de $f$ não precisa estar no domínio de $f$. Mediante essa observação, fica a questão: Por que os estudantes desenvolvem a técnica de substituição? Seria essa prática uma consequência de insuficiências praxeológicas locais? A praxeologia local é definida por Chevallard $(1999,2014)$ como sendo aquela envolvendo técnicas, justificações e explicações de um discurso matemático. Acreditamos que 
a articulação entre as variáveis visuais e o discurso tecnológico utilizado no encaminhamento do problema proposto melhora o entendimento do que se pretende construir. Com essa observação, queremos enaltecer o valor didático do software Geogebra usado nesse experimento: sua riqueza na dinamicidade. Não obstante, estamos conscientes de que esse recurso poderia ter sido usado de melhor maneira do que esta que destacamos na nossa experiência.

\section{Conclusão}

Consideramos a mediação com o recurso computacional como forma de proporcionar uma imagem muito significativa para levar os estudantes a terem algum crédito do resultado obtido, pois no ponto $x=0$, tivemos o declive da reta tangente ao gráfico de $f$ igual a zero e a reta tangente e seu declive estavam visíveis. Enaltecemos ainda o valor didático das variáveis cor, espessura e animação desse software, permitindo uma representação com sentido dos objetos não ostensivos subjacentes.

Outra representação gráfica que se revelou útil na discussão foi a da função $f(x)=$ $\operatorname{sen}\left(\frac{1}{x}\right)$, já que, por meio da imagem visual, dissipou-se o equívoco de grande parte dos estudantes, pensando que quando $x$ tendesse a zero, $f(x)$ tendia a mais infinito.

Além disso, com a imagem gráfica presente, foi possível argumentar que o produto de um número entre -1 e 1 , por 0 era igual a zero. Reconhecemos, contudo, que esta última imagem teria sido mais efetiva se se plotasse junto todo produto $x \cdot \operatorname{sen}\left(\frac{1}{x}\right)$, como indicamos na figura a seguir:

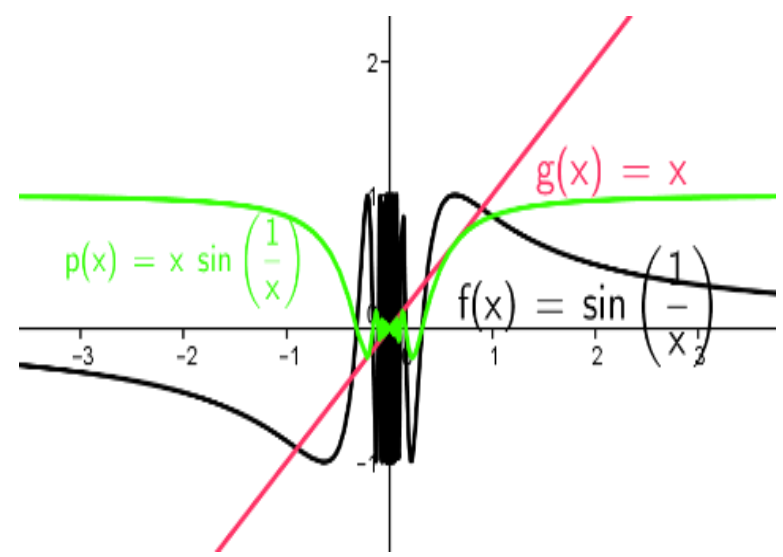

Figura 6 - Gráficos de funções fatores e da função produto Fonte: $\mathrm{O}$ autor (2014) 
Talvez com esta visualização, ficaria mais evidente que $\lim _{x \rightarrow 0} x \operatorname{sen}\left(\frac{1}{x}\right)=0$ e assim poderíamos concluir que $f^{\prime}(0)=0$.

Consideramos que a discussão tenha sido efetiva, pois oportunizou aos estudantes agirem sobre o conteúdo em causa e, dessa ação, foram sendo constatadas as suas relações pessoais com o objeto matemático visado, algumas das quais não conformes às relações institucionais vigentes.

Nesses casos, buscou-se ultrapassar os equívocos, o que julgamos ter surtido efeitos, pois os argumentos trazidos foram logicamente construídos e os estudantes mostraram ter conquistado um discurso apropriado ao que estava sendo discutido.

Na visão de Korner (1985), diríamos que houve efetivamente uma fase de construção de conhecimentos, pois o autor explica que construir um conceito é proporcionar-lhe um objeto de referência, e, na discussão havida, as intervenções dos estudantes estão carregadas desse conteúdo. No caso, diríamos que a articulação entre os ostensivos algébrico e gráfico, na discussão, oportunizou a criação de imagens mentais importantes para o desenvolvimento dos conceitos e noções associados à introdução do Cálculo Diferencial e Integral, como a utilização do Teorema do Confronto para justificar a derivabilidade da função dada no ponto $\mathrm{x}=0$.

Na ótica de Rabardel (1995, 2002), diríamos que a discussão com recurso ao instrumento proporcionou a reconstrução da relação pessoal dos estudantes quanto ao teorema em discussão.

Finalmente, consideramos pertinente destacar o efeito positivo da ferramenta computacional na conduta matemática dos estudantes, pois as afirmações deles, que aparecem na Figura 4, são exemplos de uma mudança qualitativa do pensamento matemático daqueles que participaram ativamente da pesquisa.

\section{Referências}

ARTIGUE, M. Notas do Seminário da EAE - Escola de Altos Estudos da CAPES, UNIBAN, 2010.

ÁVILA, G. Análise Matemática para Licenciatura. 2. ed. São Paulo: Editora Edgard Blucher Ltda, 2005.

BOSCH, M.; FONSECA, C.; GASCÓN, J. H. Incompletud de las Organizacioes Matematicas Locales en las Instittuciones Escolares. Recherches en Didactique des Mathématique, Grenoble, v. 24, n. 23, p. 205-250, 2004. 
CHEVALlARD, Y. Théorie Anthropologique du Didactique \& Ingénierie Didactique du Développement. Journal du seminaire tad/idd, UMR ADEF, v. 1, n. 3, p. 1-73, 2014.

CHEVALLARD, Y. Organiser l'étude 3. Ecologie \& Regulation, 2002. Disponível em: $<$ http://yves.ch evallard.free.fr/spip/spip/>. Acesso em: 10 ago. 2016.

CHEVALLARD, Y. El análisis de las prácticas docentes en la teoría antropológica de lo didáctico. Recherches en didactique des mathématiques, Grenoble, v. 19, n. 2, p. 221-266, 1999.

HITT, F. Construction of mathematical knowledge using graphic calculators (CAS) in the mathematics classroom. International Journal of Mathematical Education in Science and Technology, Waynesville, v. 42, n. 6, p. 723-735, 2011.

GUIDORIZZI, H. L. Um curso de Cálculo. v. 1, 5. ed. Rio de Janeiro: LTC, 2008.

KORNER, S. Uma introdução à Filosofia da Matemática. 1. ed. Rio de Janeiro: Zahar Editores, 1985.

LÜDKE, M.; ANDRÉ, M. E. D. A. Pesquisa em educação: abordagens qualitativas. 1. ed. São Paulo: EPU, 1986.

RABARDEL, P. People and technology a cognitive approach to contemporary instruments. Traduzido do texto francês: Les hommes et les technologies, une approche cognitive des instruments contemporains. Tradução de Heidi Wood. 1. ed. Paris: Armand Colin, 2002.

RABARDEL, P. Les hommes et les technologies, une approche cognitive des instruments contemporains. 1. ed. Paris: Armand Colin, 1995.

THURSTON, W. P. Preuve et progress en Mathématiques. REPERES-IREM , Grenoble, v. 21, p. 7 $25,1995$.

Submetido em 15 de Junho de 2017. Aprovado em 16 de Janeiro de 2018. 